\title{
A Highly Therapy-Resistant Case of B-Cell Lymphoma, Unclassifiable, with Features Intermediate between Diffuse Large B-Cell Lymphoma and Burkitt Lymphoma Showing Strong BCL2 Staining, Otherwise Indistinguishable from Burkitt Lymphoma
}

\author{
Naoki Kurita, ${ }^{1)}$ Hidekazu Nishikii, ${ }^{1)}$ Rie Nakamoto, ${ }^{1)}$ Naoya Nakamura, ${ }^{2)}$ \\ Yuzuru Kondo, ${ }^{3)}$ Yasushi Okoshi, ${ }^{1)}$ Kazumi Suzukawa, ${ }^{1)}$ Yuichi Hasegawa, ${ }^{1}$ \\ Yasuhisa Yokoyama, ${ }^{1)}$ Masayuki Noguchi, ${ }^{3)}$ and Shigeru Chiba ${ }^{1)}$
}

B-cell lymphoma, unclassifiable, with features intermediate between diffuse large B-cell lymphoma and Burkitt lymphoma (iDLBCL/BL) is a newly categorized lymphoma in the WHO Classification of Tumors of Haematopoietic and Lymphoid Tissues v4. This lymphoma has characteristics of both DLBCL and BL, morphologically and genetically. We report a case with therapy-resistant iDLBCL/BL, which used to be categorized as BL in the WHO Classification v3. This case showed strong BCL2 staining, otherwise exhibiting features of BL. The case was treated with intensive chemotherapy containing high-dose methotrexate, cyclophosphamide, and cytarabine. However, soon after the chemotherapy, the lymphoma relapsed in the central nervous system and was resistant to whole-brain radiation therapy and allogeneic stem cell transplantation. The patient died 37 days after the transplantation. The clinical course was different from that of typical BL in terms of resistance to intensive chemotherapy, in agreement with the characteristics of iDLBCL/BL. $〔 J$ Clin Exp Hematopathol 51(1) : 37-42, 2011〕

Keywords: diffuse large B-cell lymphoma, Burkitt lymphoma, intermediate diffuse large B-cell lymphoma/Burkitt lymphoma, $c-M Y C, B C L 2$

\section{INTRODUCTION}

Burkitt lymphoma (BL) is a highly aggressive nonHodgkin lymphoma often presenting at extranodal sites such as central nervous system (CNS) and bone marrow. ${ }^{1}$ The lymphoma is composed of a monotonous growth pattern of

\footnotetext{
Received : December 21, 2009

Revised : July 17, 2010

Accepted : August 26, 2010

${ }^{1)}$ Department of Hematology, Graduate School of Comprehensive Human Sciences, University of Tsukuba, Ibaraki, Japan

${ }^{2)}$ Department of Pathology, Tokai University School of Medicine, Isehara, Kanagawa, Japan

${ }^{3)}$ Department of Pathology, Graduate School of Comprehensive Human Sciences, University of Tsukuba, Ibaraki, Japan

This manuscript is based on a study that was previously reported in Medical Practice (27: 342-7, 2010) and permission for republication was obtained from the editor-inchief of Medical Practice.

Address correspondence and reprint requests to: Shigeru Chiba, M.D., Ph.D., Department of Hematology, University of Tsukuba, 1-1-1, Tennodai, Tsukuba, Ibaraki 305-8575, Japan

E-mail : schiba-t@md.tsukuba.ac.jp
}

medium-sized cells. The tumor has an extremely high proliferation fraction as well as high proliferation of apoptosis, which is characterized by a starry sky appearance, and characteristically carries a reciprocal translocation involving the $c$ MYC proto-oncogene, such as $\mathrm{t}(8 ; 14)$ or $\mathrm{t}(2 ; 8) .{ }^{2}$ Diffuse large B-cell lymphoma (DLBCL) is the most common lymphoma in adults and comprises a biologically and clinically heterogeneous category of lymphomas showing a diffuse growth pattern of large lymphoid cells. This heterogeneity is most likely caused by the variation in the genetic abnormalities in this disease. ${ }^{3}$ The World Health Organization (WHO) Classification of Tumor of Haematopoietic and Lymphoid Tissues v4 has introduced a new category of B-cell lymphoma, unclassifiable, with features intermediate between diffuse large B-cell lymphoma and Burkitt lymphoma (iDLBCL/BL), which demonstrates overlapping morphological and genetic features of DLBCL and BL. ${ }^{4}$

We report a case of a 42-year-old Japanese male with therapy-resistant iDLBCL/BL, which used to be categorized as BL in the WHO Classification v3. BCL2 was strongly 
positive, which was not typical of BL. The case was treated with intensive chemotherapy containing high-dose methotrexate, cyclophosphamide, and cytarabine, but early relapse in CNS occurred and resistance to whole-brain radiation therapy and allogeneic stem cell transplantation was exhibited.

\section{CASE REPORT}

A 42-year-old Japanese male was admitted to our hospital with submaxillary tumor and pain in the bilateral legs. Enhanced computed tomography revealed multiple masses in the left submaxilla (Fig. 1a), the mediastinum (Fig. 1b), and the abdominal cavity (Fig. 1c), to all of which ${ }^{67} \mathrm{Ga}$ was markedly accumulated. The tumors infiltrated the right, atrium of the heart and the vertebral canal. A gastric tumor was detected by gastric endoscopy (Fig. 1d). Atypical lymphocytes were found in the cerebrospinal fluid, but not in the bone marrow. Lactate dehydrogenase $(1,004 \mathrm{U} / \mathrm{L}$; normal 124-232 U/L) and soluble interleukin-2 receptor $(1,596 \mathrm{U} /$ $\mathrm{mL}$; normal 190-650 U/mL) were elevated. Biopsy of the cervical lymph node was performed. Histologically, the tumor consisted of monotonous proliferation of medium-sized lymphocytes accompanied by scattered tingible body macrophages, which gave the tumor a starry sky appearance (Fig. 2a). Immunohistochemically, the tumor cells were positive for CD20 (Fig. 2b) and CD10 (Fig. 2c), but negative for MUM1. The proliferation index assessed by Ki67 was more than $95 \%$ (Fig. 2e). Fluorescence in situ hybridization analysis revealed fusion between heavy chain of immunoglobulin $G$ $(\operatorname{IgH})$ and $c-M Y C$ genes $(\operatorname{IgH} / c-M Y C$, Fig. $2 \mathrm{~h})$, but not between $\operatorname{IgH}$ and $B C L 2$ (IgH/BCL2). G-banding karyotype analysis was not performed because sufficient specimens were not available. Although these findings were consistent with typical BL, we diagnosed iDLBCL/BL because of strong staining of BCL2 (Fig. 2d). BCL6 and Epstein-Barr virusencoded small RNA (Fig. 2g) were negative. The morphology and the absence of terminal deoxynucleotidyl transferase (Fig. 2f) did not suggest lymphoblastic lymphoma. ${ }^{5}$ The clinical stage (CS) was defined as CS IV according to the modified Ann Arbor staging system. After chemotherapy
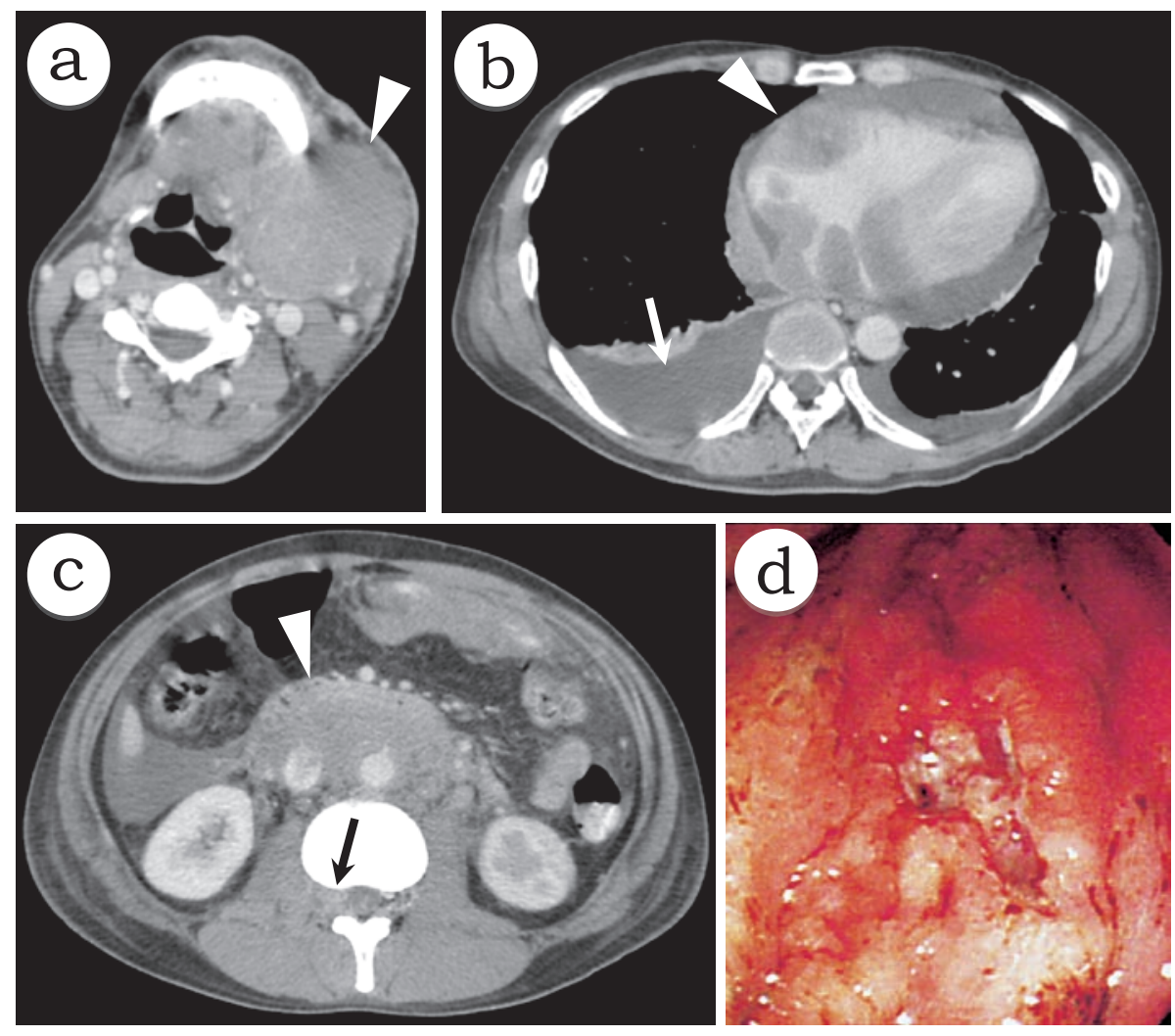

Fig. 1. Images of enhanced computed tomography and gastric endoscopy. (1a) A tumor of $6 \mathrm{~cm}$ (arrowhead) in the mandible involved the parotid gland and the submandibular glands. (1b) Tumors invaded the right atrium (arrowhead). Pleural effusion was detected (arrow). (1c) A tumor expanded around the abdominal aorta (arrowhead), which invaded to the vertebral canal through L2 intervertebral foramen (arrow). (1d) A gastric tumor was detected by gastric endoscopy. 

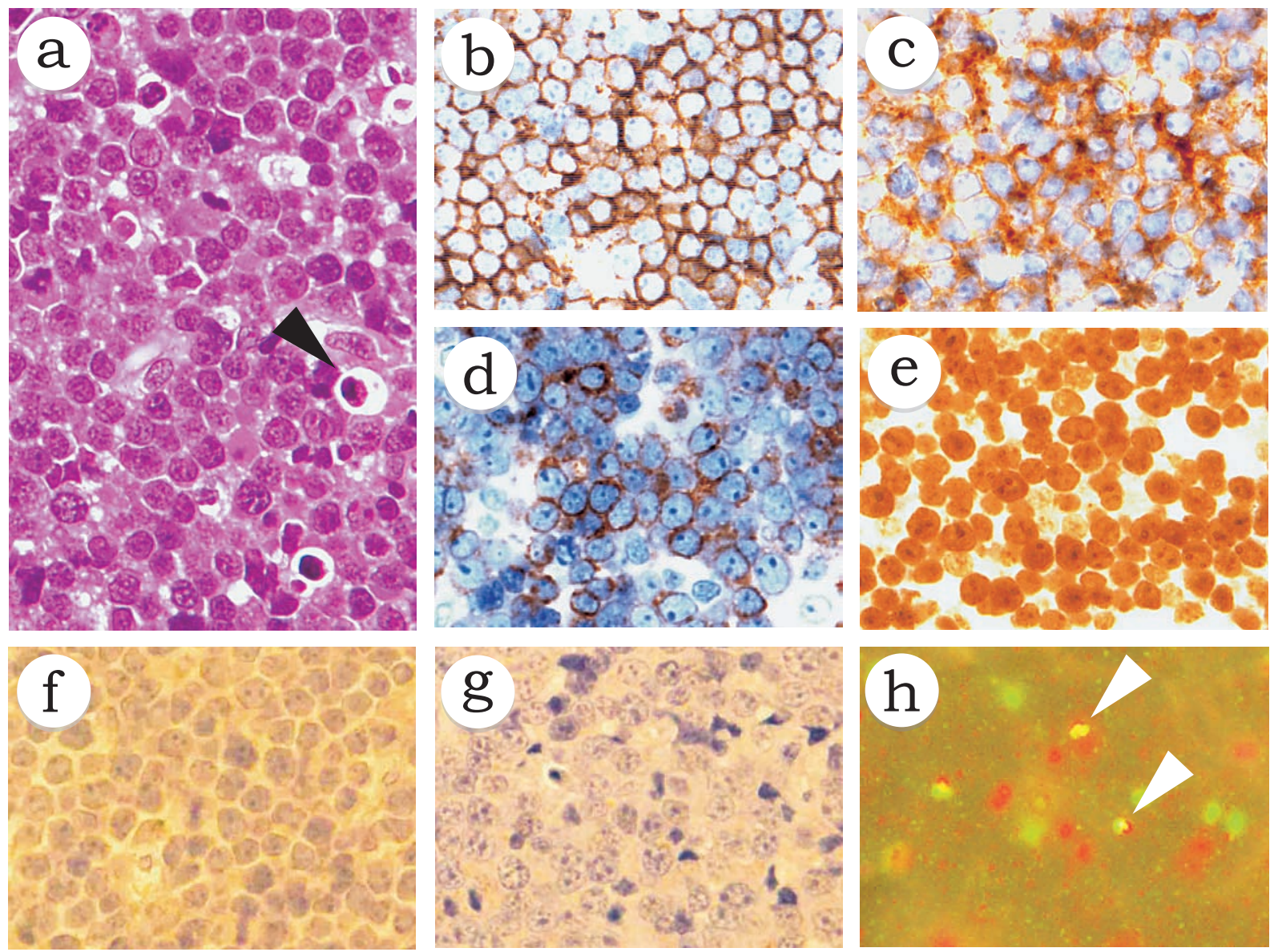

Fig. 2. Histopathology of the cervical lymph node. (2a) Hematoxylin \& eosin staining showed monotonous proliferation of medium-sized lymphocytes accompanied by a starry sky appearance (arrowhead). (2b-2f) Immunostaining of the cervical lymph node revealed that the lymphoma cells were positive for CD20 (2b), CD10 (2c), and BCL2 (2d). More than $95 \%$ of the lymphoma cells were Ki67-positive (2e). Terminal deoxynucleotidyl transferase was negative (2f). (2g) In situ hybridization of Epstein-Barr virus-encoded small RNA was negative. (2h) Fluorescence in situ hybridization analysis revealed fusion (arrowhead) between heavy chain of immunoglobulin $G$ and $c-M Y C$ genes.

with cyclophosphamide, doxorubicin, vincristine, and prednisone (CHOP), a modified LMB regimen containing highdose methotrexate (MTX), cyclophosphamide, and cytarabine was performed. ${ }^{6}$ Intrathecal chemotherapy (MTX $15 \mathrm{mg}$, cytarabine $40 \mathrm{mg}$, and prednisone $20 \mathrm{mg}$ ) was also performed 6 times because of the meningeal involvement. Multiple masses vanished and laboratory data were normalized when the protocol, divided into 3 phases containing $13 \mathrm{~g} / \mathrm{m}^{2}$ MTX in total, was completed as planned, and we judged the disease to be in complete remission because no lymphoma lesion was detected by positron emission tomography (Fig. 3) and contrasted magnetic resonance imaging (MRI). After two months, however, visual disturbance and headache appeared and MRI of the brain revealed CNS relapse in cerebellum (Fig. 4). The induction phases of the same protocol were repeated twice (containing $10 \mathrm{~g} / \mathrm{m}^{2}$ MTX in total) and wholebrain radiotherapy (30 Gy) was performed. Second complete remission was achieved and allogeneic hematopoietic stem cell transplantation using a conditioning regimen with cyclophosphamide and total body irradiation was performed three months after the relapse. Engraftment was achieved on day 16 but sudden consciousness disturbance appeared on the same day. MRI of the brain demonstrated a tumor in the right occipital lobe (Fig. 5a, 5b) indicating a second relapse. Despite discontinuation of an immunosuppressant in order to enhance graft-versus-lymphoma effect, ${ }^{7-9}$ the right occipital lobe tumor grew and extended to the cerebellum, and the patient died on transplantation day 37. Autopsy revealed that the lymphoma had infiltrated the right occipital lobe and the subarachnoid cisterns of the whole CNS (Fig. 5c-5e). 


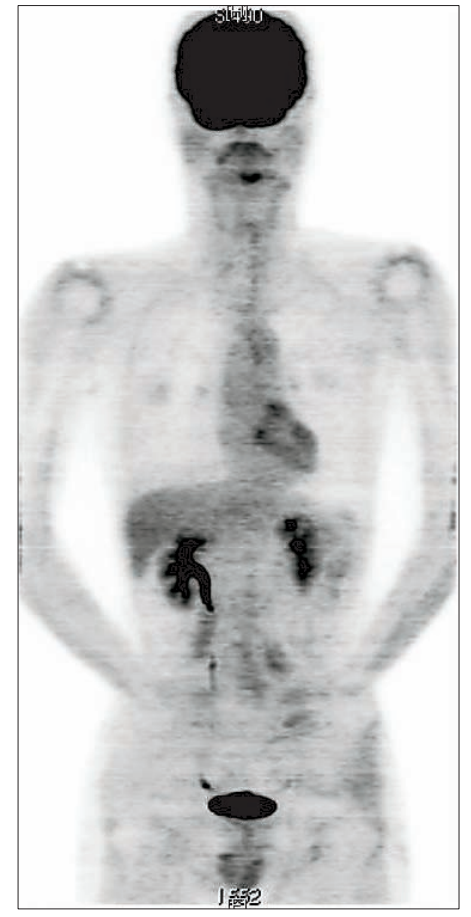

Fig. 3. Positron emission tomography after the induction therapy and 3 phases of high-dose methotrexate therapy. No lymphoma lesion was detected.

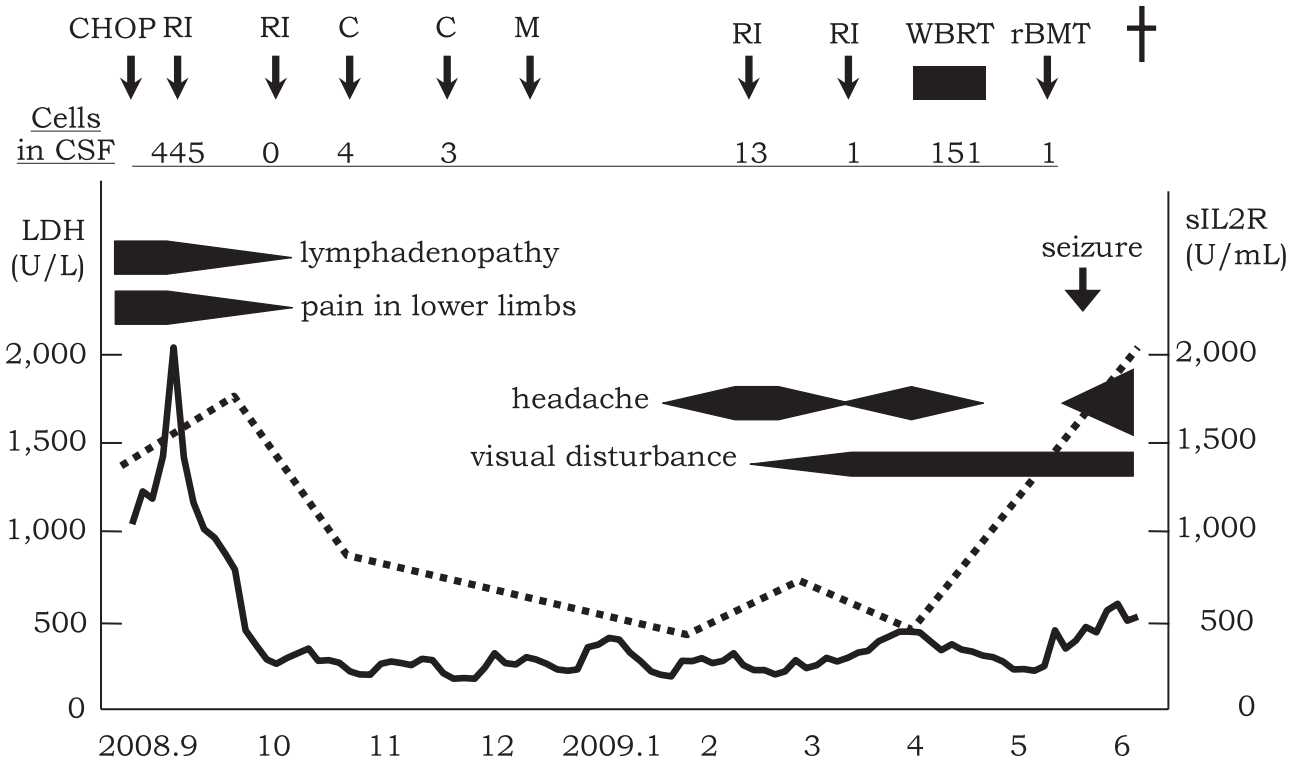

Fig. 4. Clinical course. The solid line and the dotted line show serum levels of lactate dehydrogenase (LDH) and soluble interleukin-2 receptor (sIL2R), respectively. RI, remission induction ; $\mathrm{C}$, consolidation; $\mathrm{M}$, maintenance; WBRT, whole-brain radiation therapy; rBMT, related bone marrow transplantation; CFS, cerebrospinal fluid. 

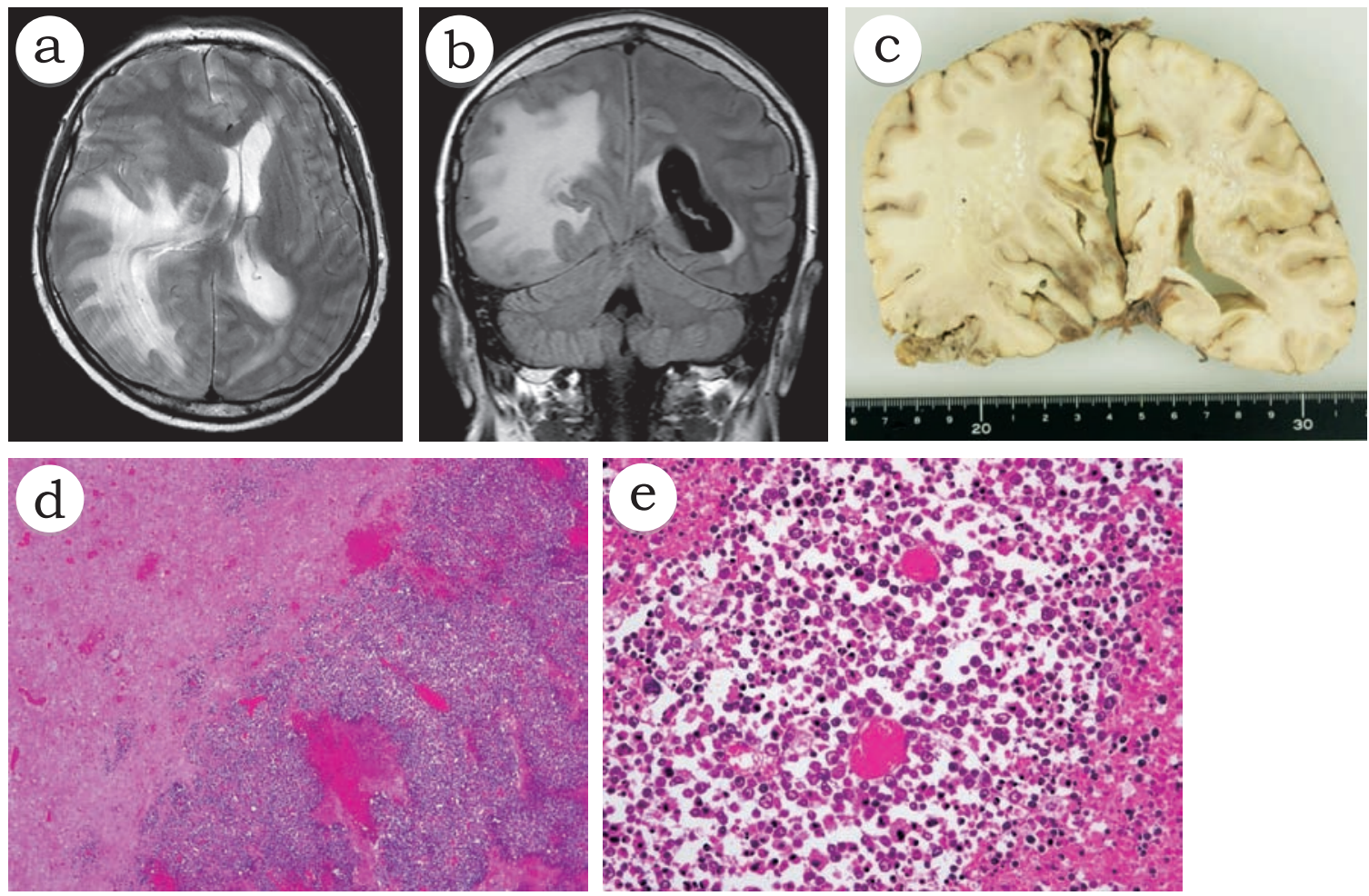

Fig. 5. The lymphoma infiltration of the central nervous system. $(\mathbf{5} \boldsymbol{a}, \mathbf{5} \boldsymbol{b})$ Magnetic resonance imaging revealed regrowth of the tumor in the right occipital lobe. Midline shift and marked cerebral edema were seen. $(\mathbf{5 c}-5 \boldsymbol{e})$ The macroscopic findings $(5 c)$ and hematoxylin \& eosin staining $(5 d-5 e)$ of the brain autopsy specimen revealed that the lymphoma had infiltrated the right occipital lobe and the subarachnoid cisterns.

\section{DISCUSSION}

On the basis of a previous version of WHO classification, the current case may have been diagnosed as BL because typical morphology with starry sky appearance, high proliferation rate of lymphoma cells (Ki67-positive cells $>90 \%$ ), and $I g H / c-M Y C$ rearrangement were all demonstrated. This case, however, showed strong staining of BCL2, being defined as iDLBCL/BL but not BL according to the WHO Classification v4.

The incidence of lymphomas with concurrent deregulation of BCL2 and c-MYC was estimated to be $4 \%$ of aggressive lymphoma cases. These lymphomas are heterogeneous in morphology, clinical presentation, and outcome. ${ }^{10} c-M Y C$, the rearrangement of which characterizes $\mathrm{BL}$, is a protooncogene that normally induces cell proliferation while also inducing apoptosis. ${ }^{11}$ The induction of apoptosis by c-MYC is thought to provide the host with a self-safeguard system against cancer, whereby c-MYC eliminates cells that accumulate its own DNA damage at abnormally high levels. ${ }^{12}$ BCL2 is an antiapoptotic protein, having a particular prosurvival role in MYC-driven tumors. It was shown in mice that BCL2 suppresses DNA repair by an unknown mechanism involving c-MYC, which results in an accumulation of DNA damage and increased tumorigenicity. ${ }^{13}$ BCL2 also has a function that inhibits c-MYC-mediated apoptosis. ${ }^{12}$ Such harmonization of molecular abnormalities might explain why double-hit lymphomas, having both $I g H / c-M Y C$ and $I g H / B C L 2$ rearrangements, are extremely chemoresistant. Indeed, doublehit cases usually present with advanced clinical stages frequently accompanied by extranodal (93\%) and CNS involvement (56\%). The median survival and 1-year survival rate were found to be only 6 months and $22 \%$, respectively. ${ }^{14}$ The strong expression of BCL2 by an unknown mechanism other than $\mathrm{IgH} / \mathrm{BCL} 2$ rearrangement, as seen in the current case, may have similar significance.

An appropriate therapeutic approach for $\mathrm{iDLBCL} / \mathrm{BL}$ is yet to be established. Relatively low-intensity and longduration chemotherapeutic strategies based on CHOP or CHOP-like regimens, standard for DLBCL in general, have proved to result in early relapse for double-hit DLBCL, ${ }^{15}$ as is the case for typical BL. Therefore, more intensive therapeutic strategies such as CODOX-M/IVAC (including cyclophosphamide, doxorubicin, vincristine, high-dose MTX, ifosfamide, etoposide, cytarabine, and vincristine) ${ }^{16}$ that can cure most BL cases have been applied to double-hit DLBCL or iDLBCL/BL. Even these strategies, however, appear to result in poorer outcome in iDLBCL/BL compared with that in BL. 
The current case could be typical of iDLBCL/BL in this regard. In such cases, allogeneic stem cell transplantation after achieving first remission may be among the most important options, although its efficacy remains to be elucidated.

It is likely that iDLBCL/BL cases were included in those that were diagnosed as typical BL but had poor prognosis. The definition of iDLBCL/BL in the new version of the WHO classification, which is based on molecular abnormalities in addition to morphology, is certainly appropriate.

\section{REFERENCE}

1 Blum K, Lozanski G, Byrd J: Adult Burkitt leukemia and lymphoma. Blood 104:3009-3020, 2004

2 Dalla-Favera R, Bregni M, Erikson J, Patterson D, Gallo R, et al. : Human c-myc oncogene is located on the region of chromosome 8 that is translocated in Burkitt lymphoma cells. Proc Natl Acad Sci USA 79:7824-7827, 1982

3 Abramson J, Shipp M: Advances in the biology and therapy of diffuse large B-cell lymphoma: moving toward a molecularly targeted approach. Blood 106:1164-1174, 2005

4 Kluin PM, Raphaël M, Harris NL, Campo E, Stein H, et al. : Bcell lymphoma, unclassifiable, with features intermediate between diffuse large B-cell lymphoma and Burkitt lymphoma. In: Swerdlow SH, Campo E, Harris NL, Jaffe ES, Pileri SA, et al. (eds): World Health Organization Classification of Tumours, WHO Classification of Tumours of Haematopoietic and Lymphoid Tissues. 4th ed, International Agency for Research on Cancer (IARC), Lyon, pp. 265-266, 2008

5 Greer W, Lee C, Callanan M, Zayed E, Sadek I: Case of acute lymphoblastic leukemia presenting with $\mathrm{t}(14 ; 18) / B C L 2, \mathrm{t}(8 ; 14) /$ $c M Y C$, and $\mathrm{t}(1 ; 2) / F C G R 2 B$. Am J Hematol 74:112-118, 2003

6 Divine M, Casassus P, Koscielny S, Bosq J, Sebban C, et al. : Burkitt lymphoma in adults: a prospective study of 72 patients treated with an adapted pediatric LMB protocol. Ann Oncol $16: 1928-1935,2005$
7 Grigg A, Seymour J: Graft versus Burkitt's lymphoma effect after allogeneic marrow transplantation. Leuk Lymphoma 43:889-892, 2002

8 Ungkanont A, Mongkonsritrakoon W, Jootar S, Srichaikul T: Allogeneic stem cell transplantation in a patient with refractory Burkitt's lymphoma using non-myeloablative conditioning regimen. Bone Marrow Transplant 26:1351-1354, 2000

9 Weinthal J, Goldman S, Lenarsky C: Successful treatment of relapsed Burkitt's lymphoma using unrelated cord blood transplantation as consolidation therapy. Bone Marrow Transplant 25:1311-1313, 2000

10 Johnson N, Savage K, Ludkovski O, Ben-Neriah S, Woods R, et al.: Lymphomas with concurrent BCL2 and MYC translocations: the critical factors associated with survival. Blood 114:2273-2279, 2009

11 Evan G, Wyllie A, Gilbert C, Littlewood T, Land H, et al. : Induction of apoptosis in fibroblasts by c-myc protein. Cell 69:119-128, 1992

12 Patel J, McMahon S: BCL2 is a downstream effector of MIZ-1 essential for blocking c-MYC-induced apoptosis. J Biol Chem 282:5-13, 2007

13 Jin Z, May W, Gao F, Flagg T, Deng X: Bcl2 suppresses DNA repair by enhancing $c-M y c$ transcriptional activity. J Biol Chem 281:14446-14456, 2006

14 Tomita N, Tokunaka M, Nakamura N, Takeuchi K, Koike J, et al. : Clinicopathological features of lymphoma/leukemia patients carrying both $B C L 2$ and $M Y C$ translocations. Haematologica 94:935-943, 2009

15 Niitsu N, Okamoto M, Miura I, Hirano M: Clinical features and prognosis of de novo diffuse large B-cell lymphoma with $\mathrm{t}(14: 18)$ and 8q24/c-MYC translocations. Leukemia 23:777-783, 2009

16 Mead GM, Sydes MR, Walewski J, Grigg A, Hatton CS, et al. (UKLG LY06 collaborators): An international evaluation of CODOX-M and CODOX-M alternating with IVAC in adult Burkitt's lymphoma: results of United Kingdom Lymphoma Group LY06 study. Ann Oncol 13:1264-1274, 2002 